\title{
Charge stripping of ${ }^{238} \mathrm{U}$ ion beam by helium gas stripper
}

\author{
H. Imao, H. Okuno, H. Kuboki, S. Yokouchi, N. Fukunishi, O. Kamigaito, H. Hasebe, T. Watanabe, \\ Y. Watanabe, M. Kase, and Y. Yano \\ RIKEN Nishina Center for Accelerator-Based Science, Wako, Saitama 351-0198, Japan
}

(Received 27 July 2012; published 11 December 2012)

\begin{abstract}
Development of a nondestructive, efficient electric-charge-stripping method is a key requirement for next-generation high-intensity heavy-ion accelerators such as the RIKEN Radioactive-Isotope Beam Factory. A charge stripper employing a low- $Z$ gas is an important candidate applicable to high-intensity uranium beams for replacing carbon-foil strippers. In this study, a high-beam-transmission chargestripping system employing helium gas for ${ }^{238} \mathrm{U}$ beams injected at $10.8 \mathrm{MeV} / \mathrm{u}$ was developed and demonstrated for the first time. The charge-state evolution measured using helium in a thickness range of $0.24-1.83 \mathrm{mg} / \mathrm{cm}^{2}$ is compared with theoretical predictions. Energy attenuation and energy spread due to the helium stripper are also investigated.
\end{abstract}

DOI: 10.1103/PhysRevSTAB.15.123501

PACS numbers: $25.70 . \mathrm{Kk}, 29.20 .-\mathrm{c}, 34.50 . \mathrm{Fa}$

\section{INTRODUCTION}

\section{A. Charge strippers at RIBF}

A critical issue at the RIKEN Radioactive-Isotope Beam Factory (RIBF) [1] is the need to improve the intensity of ${ }^{238} \mathrm{U}$ beams $(\sim 3.5 \mathrm{pnA})$ toward the intensity goal of $1 \mathrm{p} \mu \mathrm{A}$; this improvement is expected to provide a huge breakthrough for exploring new domains of the nuclear chart.

Recently, a new injector, RILAC2 [2], which has a $28-\mathrm{GHz}$ superconducting electron cyclotron resonance ion source (SC-ECRIS) [3], was successfully commissioned and operated in user runs [4]. The intensity of ${ }^{238} \mathrm{U}$ beams at the RIBF has steadily increased, mainly because of improvements in the 28-GHz SC-ECRIS [5].

The uranium beams emitted from RILAC2 are accelerated by four separate-sector cyclotrons (Fig. 1): the RIKEN ring cyclotron (RRC) [6], a fixed-frequency ring cyclotron (fRC) [7], an intermediate-stage ring cyclotron (IRC) [8], and a superconducting ring cyclotron (SRC) [9]. The accelerated beams are then delivered to the in-flight RI beam generator, BigRIPS [10].

To obtain higher-power ${ }^{238} \mathrm{U}$ beams by using a powerful injector, one must resolve a number of issues, including those related to beam acceleration, space charge effects, heat loading, and radiation damage. In particular, the development of a reliable, efficient electric-charge-stripping method that can be applied to high-intensity uranium beams is an urgent concern. The sophistication of the stripping method for such very-heavy ions at various energies has also been strongly pursued among the world's

Published by the American Physical Society under the terms of the Creative Commons Attribution 3.0 License. Further distribution of this work must maintain attribution to the author(s) and the published article's title, journal citation, and DOI. heavy-ion accelerator facilities (e.g., BNL [11], MSU [12], and GSI [13]).

In the present process of acceleration of uranium beams at the RIBF, two carbon-foil charge strippers [14] are used; one is used after the RRC, and the other is used after the fRC (Fig. 1). The first is a $0.3-\mathrm{mg} / \mathrm{cm}^{2}$ foil that converts $10.8-\mathrm{MeV} / \mathrm{u} \mathrm{U}^{35+}$ beams to $10.6-\mathrm{MeV} / \mathrm{u} \mathrm{U}^{71+}$ ones with an efficiency of about $17 \%$. The second is a $17-\mathrm{mg} / \mathrm{cm}^{2}$ foil that converts $51-\mathrm{MeV} / \mathrm{u} \mathrm{U}^{71+}$ beams to $46-\mathrm{MeV} / \mathrm{u}$ $\mathrm{U}^{86+}$ ones with an efficiency of approximately $27 \%$.

Although solid carbon-foil charge strippers provide good charge-stripping efficiency, which is assisted by density effect (e.g., the Bohr-Lindhard effect [15] and Betz-Grodzins effect [16]), two serious problems emerge particularly for the first stripper after the RRC: (1) a short usable time and (2) nonuniform thickness. The continuous

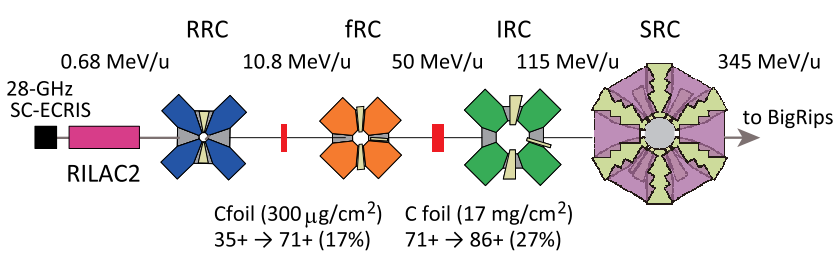

FIG. 1. Uranium beam acceleration scheme at the RIBF. $\mathrm{U}^{35+}$ beams extracted from the $28-\mathrm{GHz}$ ECRIS are accelerated to $0.68 \mathrm{MeV} / \mathrm{u}$ using RILAC2 and are then fed into the RRC. The beams are accelerated to $10.75 \mathrm{MeV} / \mathrm{u}$ in the RRC and then go through the first carbon-foil stripper $\left(0.3 \mathrm{mg} / \mathrm{cm}^{2}\right)$. A stripping efficiency of approximately $17 \%$ can be reached by selecting the charge state $\mathrm{U}^{71+}$. After the acceleration to $50 \mathrm{MeV} / \mathrm{u}$ using the $\mathrm{fRC}$, the charge state of the beams is converted using a second thick carbon-foil stripper $\left(17 \mathrm{mg} / \mathrm{cm}^{2}\right)$. The selected charge state, $\mathrm{U}^{86+}$, is obtained with an efficiency of approximately $27 \%$. The $\mathrm{U}^{86+}$ beams are subsequently accelerated to an energy of $345 \mathrm{MeV} / \mathrm{u}$ using the IRC and SRC. Finally, the beams are delivered to the in-flight RI beam generator, BigRIPS [10]. 
irradiation with uranium beams induces significant longitudinal emittance growth [17], which dominantly limits the usable time of fixed carbon-foil strippers typically as little as a half day for the present use. To improve the limited usable time, various kinds of carbon foils including polymer-coated carbon foils have been tested $[18,19]$.

The ultimate goal of the RIBF is the acceleration of $1-\mathrm{p} \mu \mathrm{A}$ uranium ion beams. The intensity of the uranium ion beam is expected to be $\sim 15 \mathrm{p} \mu \mathrm{A}$ at the first stripper, which is more than 100 times higher than the current value. The carbon-foil stripper will not tolerate such highintensity beams, which deposit subkilowatt powers, without drastic improvements.

\section{B. Low- $Z$ gas stripper}

Gas strippers are important candidates for replacing carbon-foil strippers for application to high-intensity ${ }^{238} \mathrm{U}$ beams in the future because of their potential high durability and uniform thickness. However, the measured equilibrium charge state is lower; it is 57+ for Ar and 56+ for $\mathrm{N}_{2}$ with $10.8-\mathrm{MeV} / \mathrm{u}{ }^{238} \mathrm{U}$ beams [20] compared with $72+$ for carbon-foil strippers. The reason is the absence of the density effect. The mean charge state of $69+$, which is the minimum acceptable charge state of the present fRC at the RIBF, is expected to be obtained via $\mathrm{N}_{2}$ gas stripping in the energy range of $24-25 \mathrm{MeV} / \mathrm{u}$ [21]. Drastic modification of the fRC or the construction of an extensive accelerator and decelerator are required for the actual application of $\mathrm{N}_{2}$ or Ar gas strippers at the RIBF.

A charge stripper employing a low- $Z$ ( $Z$ : atomic number) gas is a possible method of obtaining a higher equilibrium charge state [22]. The equilibrium charge state is determined by the competition between the projectile electron loss (EL) and electron capture (EC) processes. Because the electron capture process is suppressed, the low- $Z$ gas is expected to yield high equilibrium charge states while maintaining the intrinsic robustness of the gas. In fact, the result of a previous experiment performed at the RIBF using thin $\mathrm{He}$ gas targets $\left(\leq 0.015 \mathrm{mg} / \mathrm{cm}^{2}\right)$ indicates a high equilibrium mean charge state near $66+$ at $10.8 \mathrm{MeV} / \mathrm{u}$ [22].

Theoretically, the charge-state evolution in a dilute gas can be obtained by solving the coupled linear differential equations of the ionization-capture kinetics with chargechanging cross sections [23]. Although the low- $Z$ species $\mathrm{H}_{2}$ and $\mathrm{He}$ are simple target systems with two electrons, the theoretical EL and EC cross sections for ${ }^{238} \mathrm{U}$ with low- $Z$ gases are known only to a factor of 2-3 at best [24-26]. This is insufficient for obtaining the charge-state evolution reliably. In addition, knowledge of the degradation in beam quality due to low- $Z$ gas strippers, including energy attenuation and energy spread, is also currently insufficient for actual use. Such processes related to beam degradation depend fundamentally on the charge-state evolution of the stripping media.
In this study, the maximum mean charge state, the chargestate evolution, and the properties of the charge-stripped ${ }^{238} \mathrm{U}$ beams were investigated using $\mathrm{He}$ gas targets. We prepared a $0.5-\mathrm{m}$ windowless target section in the high-vacuum beam line by adopting high-performance differential-pumping systems at both ends of the section. A high-beam-transmission charge-stripping system dedicated to helium gas is demonstrated for the first time.

\section{HELIUM-GAS TARGET SYSTEM}

A key component for realizing a massive low- $Z$ gas charge stripper is a windowless connection between the high-vacuum beam line $\left(\approx 10^{-3} \mathrm{~Pa}\right)$ and the highpressure target region. The differential-pumping performance is reduced tremendously for $\mathrm{H}_{2}$ and $\mathrm{He}$ gas compared with that for ordinary medium- $Z$ gases such as $\mathrm{N}_{2}$.

Of the low- $Z$ gases helium and hydrogen, helium is more suitable for charge stripping of high-intensity uranium beams because of its easier accumulation, larger chargeexchange cross sections, lower energy deposition and the absence of an explosion hazard.

In a previous experiment involving the use of a differential-pumping gas-cell system (14 cm in cell length) [20,22,27], the maximum He gas thickness that could be realized while maintaining a tolerable beam line vacuum was found to be $0.015 \mathrm{mg} / \mathrm{cm}^{2}$. However, for $\mathrm{N}_{2}$ gas, a thickness of $1.1 \mathrm{mg} / \mathrm{cm}^{2}$ could easily be achieved. The lower EL and EC cross sections for ${ }^{238} \mathrm{U}$ colliding with a low- $Z$ gas, in comparison to those for ${ }^{238} \mathrm{U}$ colliding with $\mathrm{N}_{2}$, yield a larger mean-free path of the injected ions, which results in slow equilibration. A simple estimation of the charge evolution using theoretical EL and EC cross sections for $\mathrm{He}$ [22] indicates that a thickness greater than $1 \mathrm{mg} / \mathrm{cm}^{2}$ is required for $10.8-\mathrm{MeV} / \mathrm{u}{ }^{238} \mathrm{U}$ beams to attain the maximum charge state, whereas a value of only $0.4 \mathrm{mg} / \mathrm{cm}^{2}$ is required for $\mathrm{N}_{2}$ [20].

In an earlier study, a long low- $Z$ gas target system ( $8 \mathrm{~m}$ in target length) was developed for accumulating hydrogen gas to a thickness greater than $1 \mathrm{mg} / \mathrm{cm}^{2}$ [28]. A few comparative tests using the $8-\mathrm{m}$ low- $Z$ gas target system were performed in the present study. However, a shorter target system is strongly desired for actual use. We found that the target length required for accumulating up to $2 \mathrm{mg} / \mathrm{cm}^{2}$ of helium can be reduced dramatically from 8 to $0.5 \mathrm{~m}$ by optimizing the design. Such a short system is favorable for improving the beam transmission efficiency.

A short $(0.5 \mathrm{~m})$ gas target system (Fig. 2) for demonstrating such a high-beam-transmission helium gas stripper was developed in the present study. In the system, two tube-separated three-stage differential-pumping systems are used, one on either side of the target. The conductances among the vacuum chambers are limited by the diameters of the tubes, which are $6-10 \mathrm{~mm}$. A vacuum in stage 1 is generated using a powerful mechanical booster pump (EH4200; Edwards Vacuum, U.K.) that backs up a 


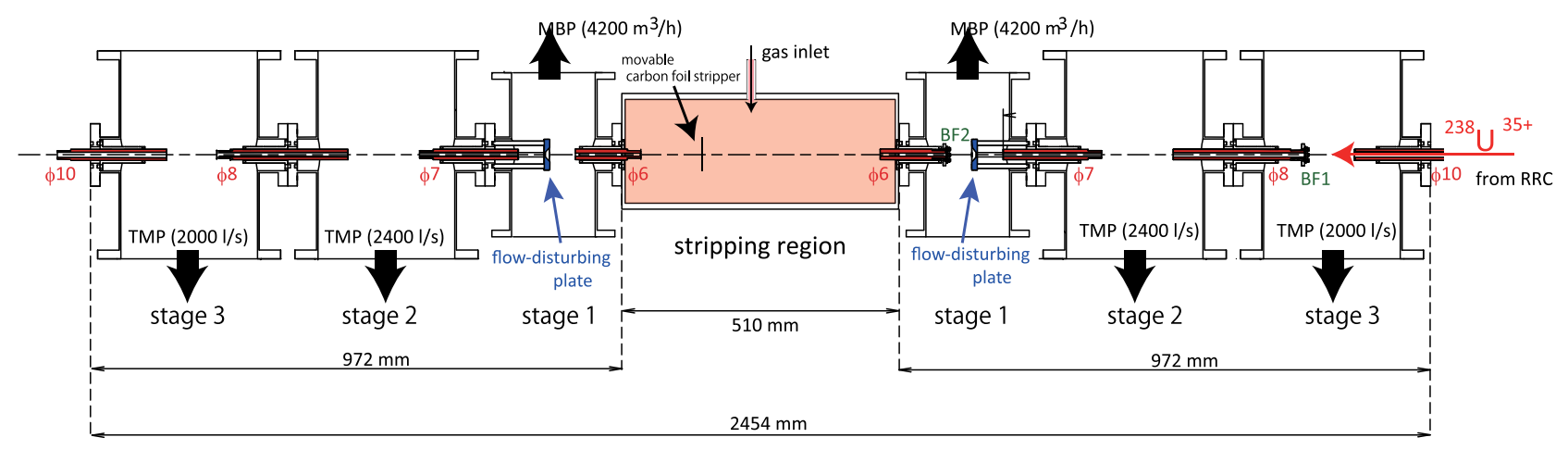

FIG. 2. Cross-sectional view of the 0.5-m charge-stripping system. The system used here consists of two three-stage differentialpumping systems. Flow-disturbing plates are furnished to decouple the gas flows between stages 1 and 2 . Beam current detectors with four segmental baffles, BF1-BF2, are attached at the beam entrance of the apertures to monitor the beam loss. The gas inlet line is connected to the gas-handling system. In addition, the target chamber is equipped with a foil changer for the carbon-foil stripper.

rotary oil pump (E2M275; Edwards Vacuum, U.K.). A high-throughput turbomolecular pump is used in stage 2 (TG2410F; Osaka Vacuum, Japan), and an ordinary one is used in stage 3 (STP-H2000C; Seiko Seiki, Japan). Flow-disturbing plates are located between the first and second stages; they were specially designed to reduce the flow of the supersonic gas jet [28]. The flow rate of helium gas introduced to the target chamber was measured and controlled by a mass flow controller (HFC-303; KOFLOC, Japan).

Although the length of the differential-pumping system was as small as $1 \mathrm{~m}$, and the diameter of the beam passage was more than $6 \mathrm{~mm}$, a pressure transition from $15 \mathrm{kPa}$ to $\sim 10^{-3} \mathrm{~Pa}$ was achieved for He. The pressures of the upstream and downstream beam lines were 1 order of magnitude lower than the pressure in the third stage. In the 0.5$\mathrm{m}$ charge-stripping system, the thickness of He was limited to $2 \mathrm{mg} / \mathrm{cm}^{2}$ to maintain a tolerable beam line pressure.

The required helium flow rate in standard liters per minute (SLM) is shown as a function of target thickness in Fig. 3. The target thicknesses were determined with the measured pressures for all chambers. The pressure distribution of helium target was calculated by using SOLIDWORKS flow simulation (software based on the finite element method, Dassault Systemes Solidworks, Concord,

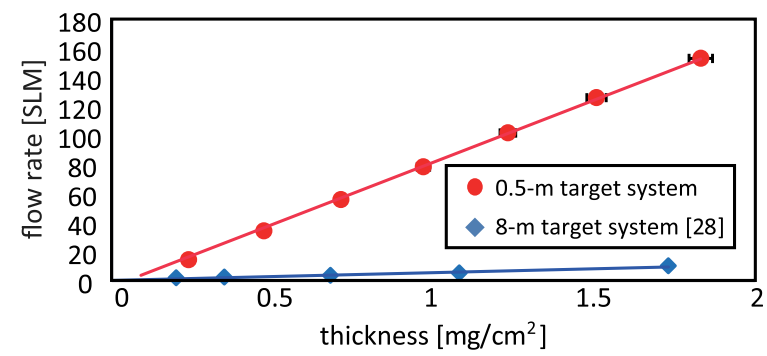

FIG. 3. Required flow rate as a function of the helium thickness for both 0.5 - and 8-m target systems.
MA, USA). In the present $0.5-\mathrm{m}$ system, the consumption of helium gas is relatively high in order to achieve a thick target. We supplied helium to the target chamber from the gas-handling system using a bundle of helium-gas cylinders $\left(7 \mathrm{~m}^{3} \times 30\right)$.

Note that heavier gaseous species such as $\mathrm{N}_{2}, \mathrm{Ne}$, and Ar can be easily confined simply by changing the gas cylinders in the present system. In addition, the present system has a foil changer for carbon-foil strippers in the target section. Comparative measurements using $\mathrm{Ne}$ gas strippers and carbon-foil strippers were performed in the present study.

\section{EXPERIMENT}

In the experiment at the RIBF, the 0.5-m helium-gas charge-stripping system was located in the beam line after the RRC (Fig. 4). The $0.68-\mathrm{MeV} / \mathrm{u} \mathrm{U}^{35+}$ beams extracted from RILAC2 were accelerated to $10.8 \mathrm{MeV} / \mathrm{u}$ using the RRC. The ${ }^{238} \mathrm{U}^{35+}$ beams emitted from the RRC (50-200 enA in these measurements) were directly injected to the $0.5-\mathrm{m}$ target system via four quadrupole triplets (QTA01-03, QTM04).

We transported the ${ }^{238} \mathrm{U}$ beams passing through the charge-stripping section with a transmission efficiency of around $80 \%$ by adjusting the magnet parameters. The downstream beam current of the charge state, selected by using a $90^{\circ}$ dipole magnet (DMM1) after the stripper, was measured using a Faraday cup (FC-M11). The magnetic field of these magnets was corrected to account for the energy loss in the gas stripper.

Three plastic scintillators (SC-H12, SC-H16, and SCK51) were used to check the kinetic energy of the beam using the time of flight between them [29]. The current beam transport system between the exit of the RRC and the entrance of the IRC is equipped with several quadrupole magnets and horizontal and vertical steering coils, which were used to optimize the beam trajectory. 


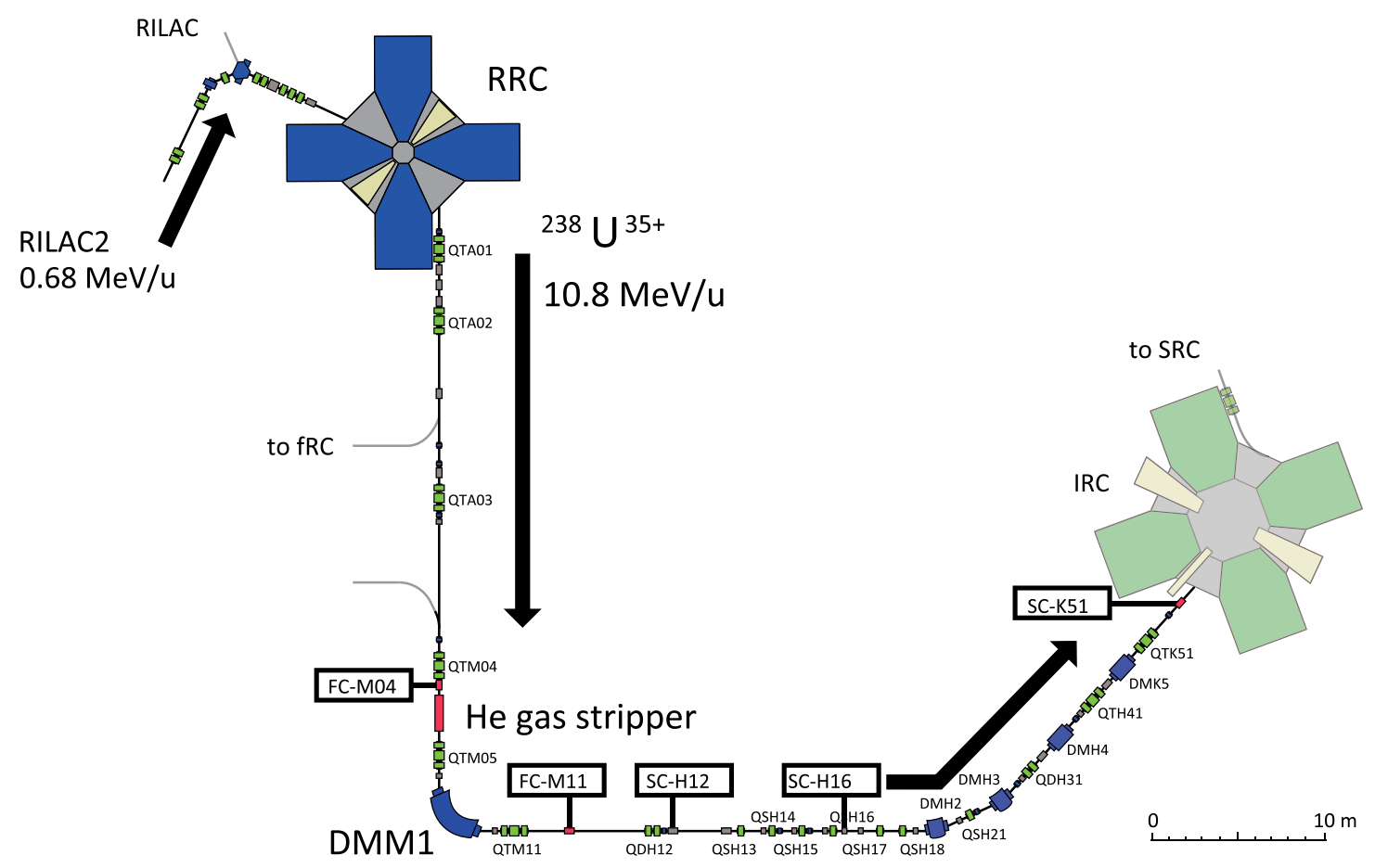

FIG. 4. Schematic view of the entire experimental setup. The RRC accelerates $\mathrm{U}^{35+}$ beams coming from RILAC2 to an energy of $10.8 \mathrm{MeV} / \mathrm{u}$. The emitted beams of around $150 \mathrm{enA}$ are fed into the He gas stripper. The charge-state distribution of the passing beams is analyzed using a dipole magnet, DMM1, and a Faraday cup, FC-M11. Plastic scintillators (SC-H12, SC-H16, and SC-K51) are used to measure the beam's kinetic energy and longitudinal distribution. Many quadrupole magnets (QS*: singlet; QD*: doublet; QT*: triplet) were used in the beam transport system to optimize the beam trajectory.

The transmission efficiency of the $0.5-\mathrm{m}$ target system was compared to that of the 8-m system (Fig. 5). Because of the short length of the target section, the transmission efficiency of the present system is considerably higher than that of the 8-m system. In these measurements, the magnet parameters of the quadrupole triplets upstream of the stripper (QTA01-03, QTM04) were fixed to the tuned values of the empty targets.

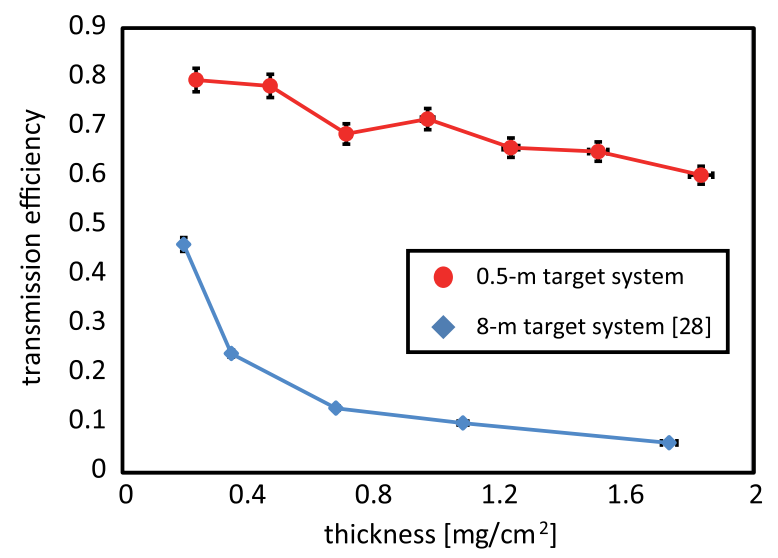

FIG. 5. Transmission efficiency of a selected charge state as a function of the helium thickness. The most probable charge state for each thickness was selected and transported for the measurements.

\section{RESULTS AND DISCUSSION}

\section{A. Charge-state evolution}

The fraction $F\left(q_{i}\right)$ of the charge state $q_{i}$ was determined using the same procedure as in previous measurements [20]. We used the measured injected beam current with FC-M04 $\left(I_{\text {M04 }}\right)$ and the analyzed one with FC-M11 $\left(I_{\text {M11 }}\right)$ to deduce $F\left(q_{i}\right)$ :

$$
F\left(q_{i}\right)=\frac{1}{S} \frac{I_{\mathrm{M} 11} / q_{i}}{I_{\mathrm{M} 04} / q_{\mathrm{ini}}}
$$

where $S=\sum_{i}\left(I_{\mathrm{M} 11} / q_{i}\right) /\left(I_{\mathrm{M} 04} / q_{\text {ini }}\right)$ and $q_{\text {ini }}$ is the initial charge state (equal to $35+$ ). We note that $I_{\mathrm{M} 04}$ was measured in every measurement of $I_{\mathrm{M} 11}$.

Gaussian functions were fitted to the obtained $F\left(q_{i}\right)$ for He gas to derive the mean charge states, $q_{\text {mean }}$, and the distribution widths, $w_{q}$ (Fig. 6). The resultant values are summarized in Table I.

The mean charge $q_{\text {mean }}$ for He strippers measured in this study is plotted as a function of the calculated gas thicknesses (Fig. 7). For comparison, plots for Ne measured in this study and Ar [20] are also displayed. The data on the charge-state evolution obtained for the present system were comparable with those obtained for the 8-m target system. The charge states for the He stripper increased gradually as the thickness increased up to a thickness of $1 \mathrm{mg} / \mathrm{cm}^{2}$ and then became saturated. The obtained maximum mean 


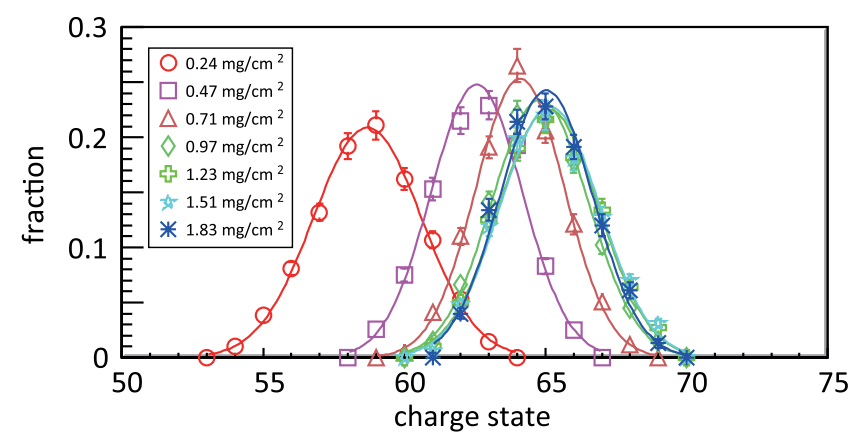

FIG. 6. Results of Gaussian fitting to the charge-state distribution for He.

charge state $(65+)$ for the He gas stripper is much higher than those obtained with other rare gases. To calculate the charge-state evolution, we used a Monte Carlo method with the EL cross sections for ${ }^{238} \mathrm{U}$ based on the binary encounter model (BEM) $\sigma_{\text {BEM }}^{\text {el }}[30]$ and Schlachter's semiempirical EC cross sections $\sigma_{\text {Sch }}^{\text {ec }}$ [31]. The effect of multiple charge transfers is negligible for $\mathrm{He}$ gas and was disregarded in this calculation. The cross section, $\sigma_{\mathrm{BEM}}^{\mathrm{el}}$, is given by

$$
\sigma_{\mathrm{BEM}}^{\mathrm{el}}=\sum_{n} N_{n} \pi\left(\frac{Z_{2} e^{2}}{U_{n}}\right)^{2} G\left(\frac{v_{p}}{v_{n}}\right)
$$

where $N_{n}, U_{n}, v_{n}$, and $v_{p}$ are the number of electrons in the $n$th shell, their binding energy, their orbital velocity, and the projectile velocity, respectively. $Z_{2}$ is the atomic number of the target, and $G$ is a function of the scaled velocity [32]. We used the data for uranium in a reference [33] for the binding energies. Schlachter's semiempirical cross section is given by

$$
\sigma_{\mathrm{Sch}}^{\mathrm{ec}}=1.1^{-8} q^{3.9} Z_{2}^{4.2} E^{-4.8},
$$

where $\sigma_{\mathrm{Sch}}^{\mathrm{ec}}$ is in $\mathrm{cm}^{2}, q$ is the ion charge, and $E$ is the projectile ion energy in $\mathrm{keV} / \mathrm{u}$. The charge-dependent energy-loss cross sections calculated using the CASP code

TABLE I. Summary of experimental conditions and chargestate distribution results for the He charge stripper. The given experimental parameters are the gas thickness, gaseous flow rate in SLM, and pressure in the stripping section. The fitting results for the measured charge-state distribution for the mean charge $q_{\text {mean }}$ and the distribution width $w_{q}(1 \sigma)$ are listed.

\begin{tabular}{lccccc}
\hline \hline Gas & $\begin{array}{c}\text { Thickness } \\
{\left[\mathrm{mg} / \mathrm{cm}^{2}\right]}\end{array}$ & $\begin{array}{c}\text { Flow rate } \\
{[\mathrm{SLM}]}\end{array}$ & $\begin{array}{c}\text { Pressure } \\
(\mathrm{P} 0)[\mathrm{kPa}]\end{array}$ & $q_{\text {mean }}$ & $w_{q}$ \\
\hline $\mathrm{He}$ & $0.24(1)$ & $16.0(2)$ & $2.51(4)$ & $58.7(1)$ & $1.92(3)$ \\
& $0.47(1)$ & $38.0(4)$ & $4.89(7)$ & $62.6(1)$ & $1.61(3)$ \\
& $0.71(1)$ & $62(1)$ & $7.2(1)$ & $64.1(1)$ & $1.57(3)$ \\
& $0.97(2)$ & $87(1)$ & $9.6(1)$ & $64.8(1)$ & $1.71(5)$ \\
& $1.23(2)$ & $113(1)$ & $12.0(2)$ & $65.1(1)$ & $1.77(5)$ \\
& $1.51(3)$ & $140(2)$ & $14.5(2)$ & $65.2(1)$ & $1.76(5)$ \\
& $1.83(4)$ & $170(2)$ & $17.1(2)$ & $65.1(1)$ & $1.64(4)$ \\
\hline \hline
\end{tabular}

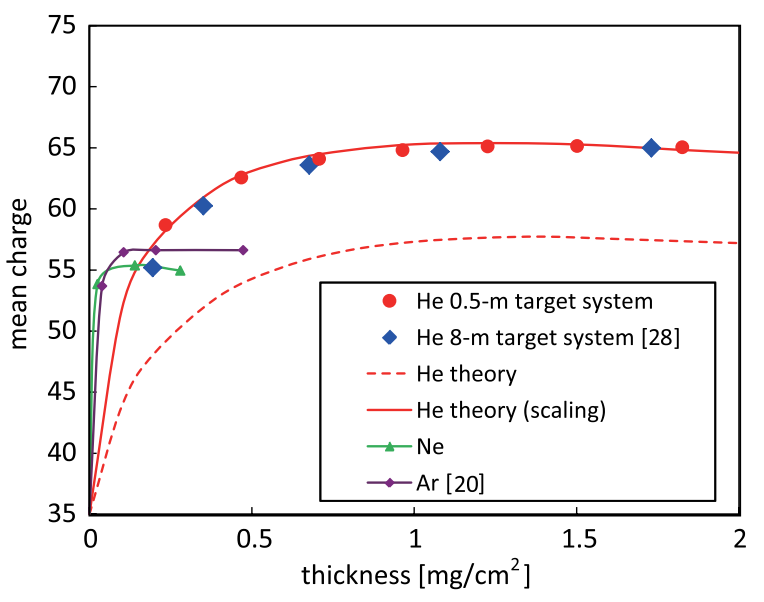

FIG. 7. Measured and calculated charge-state evolution for $\mathrm{He}$, $\mathrm{Ne}$, and $\mathrm{Ar}$ [20]. The maximum mean charge state for $\mathrm{He}$ of around $65+$ was obtained in both the $0.5-\mathrm{m}$ and $8-\mathrm{m}$ target systems. The calculation was performed using the chargechanging cross sections $\sigma_{\mathrm{BEM}}[30]$ and $\sigma_{\mathrm{Sch}}[31]$ for ${ }^{238} \mathrm{U}$, while accounting for energy attenuation in the gas stripper. In addition, the calculation with scaled cross sections is shown.

[34], which is based on the unitary convolution approximation by Grande and Schiwietz [35,36], were implemented in our simulation. In the Monte Carlo simulation code, the charge-changing and energy-loss processes were treated as uncorrelated.

The theory based on $\sigma_{\mathrm{BEM}}^{\mathrm{el}}$ and $\sigma_{\text {Sch }}^{\mathrm{ec}}$ predicts a significantly different charge-state evolution for helium (dashed line in Fig. 7). To find more suitable cross sections, the measured charge-state distributions $F_{\xi}^{\exp }\left(q_{i}\right)$ for all gas thicknesses $\xi$ were fitted using the calculated fraction function $F_{\xi}^{\mathrm{cal}}\left(q_{i}\right)$ based on the scaled cross sections. Here the scaled cross sections are defined as $\sigma_{n}^{\text {el }}\left(q_{i}, E\right)=$ $\alpha \sigma_{\mathrm{BEM}}^{\mathrm{el}}\left(q_{i}, E\right)$ and $\sigma_{n}^{\mathrm{ec}}\left(q_{i}, E\right)=\beta \sigma_{\mathrm{Sch}}^{\mathrm{ec}}\left(q_{i}, E\right)$, where $\alpha$ and $\beta$ are the scaling factors to be found from the fit, and the kinetic energy $E$ of the projectile ${ }^{238} \mathrm{U}$ is explicitly described. $F_{\xi}^{\mathrm{cal}}\left(q_{i}\right)$ is given by the solution of the rate equation,

$$
\begin{aligned}
\frac{d F\left(q_{i}, E\right)}{d x}= & n\left\{F\left(q_{i-1}, E\right) \sigma_{n}^{\mathrm{el}}\left(q_{i-1}, E\right)\right. \\
& -F\left(q_{i}, E\right)\left[\sigma_{n}^{\mathrm{el}}\left(q_{i}, E\right)+\sigma_{n}^{\mathrm{ec}}\left(q_{i}, E\right)\right] \\
& \left.+F\left(q_{i+1}, E\right) \sigma_{n}^{\mathrm{ec}}\left(q_{i+1}, E\right)\right\},
\end{aligned}
$$

where $n$ is the gas density. Further, $d E / d x$ in the gas stripper is approximated as $d E\left(q_{\text {avg }}\right) / d x$, where $q_{\text {avg }}=$ $\sum_{i} q_{i} F\left(q_{i}\right)$ is the calculated average charge state. The $\chi^{2}$ value in the fitting was determined as

$$
\chi^{2}=\sum_{j} \sum_{i}\left\{\left(F_{\xi_{j}}^{\mathrm{exp}}\left(q_{i}\right)-F_{\xi_{j}}^{\mathrm{cal}}\left(q_{i}\right) / \delta F_{\xi_{j}}^{\mathrm{exp}}\left(q_{i}\right)\right\}^{2},\right.
$$

where $\delta F_{\xi_{j}}^{\exp }$ represents the experimental errors of the measured fraction, which are caused primarily by beam 

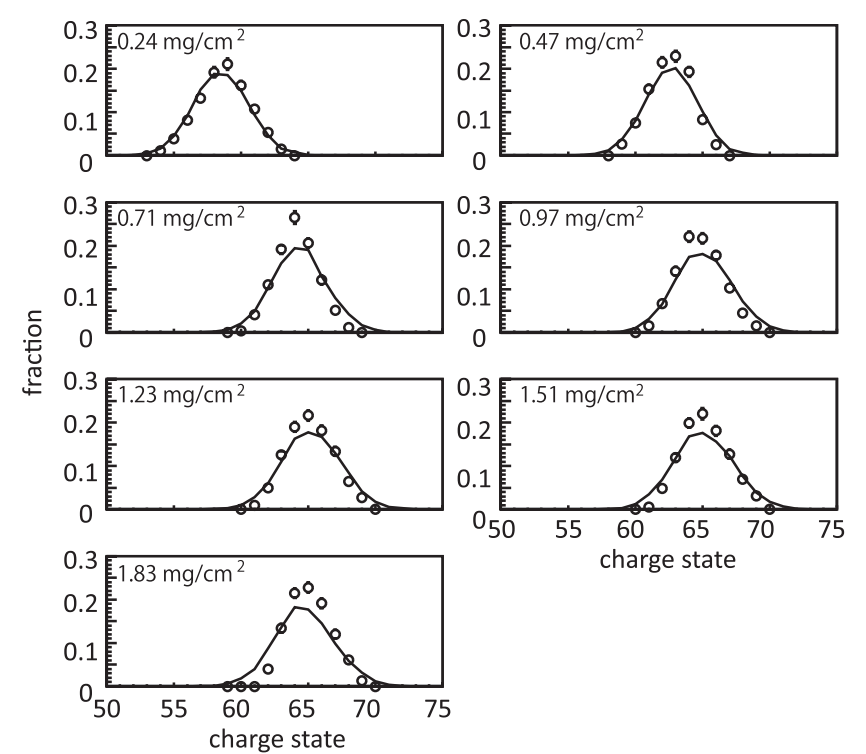

FIG. 8. Fitting results for the charge-state distribution in $\mathrm{He}$ gas strippers of various thicknesses. Solid lines indicate calculated probabilities, $F_{\xi}^{\mathrm{cal}}\left(q_{i}\right)$.

fluctuations, and $j$ is the index of the target thickness. The minimization for the least $\chi^{2}$ fitting method was performed using the MINUIT code [37]. The results of the fittings are shown in Fig. 8. The experimental charge-state distribution can be fitted well using the two parameters $\alpha$ and $\beta$. The resulting scaling factors were determined to be $\alpha_{\mathrm{He}}=$ 3.1(1) and $\beta_{\mathrm{He}}=0.62(1)$, respectively. The charge-state evolution calculated with the scaled cross sections is also shown in Fig. 7 (solid line). The higher charge state can be explained by the large scaling factors for the EL cross sections.

Note that the fraction of $64+$ is significantly enhanced for He having a thickness of $0.71 \mathrm{mg} / \mathrm{cm}^{2}$, as shown in Fig. 8. The transient enhancement is qualitatively interpreted as the effect of the electron shell of uranium on the ionization process because the charge states $63+$ and $64+$ represent the boundary between $\mathrm{M}$ - and $\mathrm{N}$-shell ionization. Furthermore, the kinetic energy of $10.8 \mathrm{MeV} / \mathrm{u}$ for uranium beams $(\beta=0.15)$ corresponds to a kinetic energy of $5.8 \mathrm{keV}$ for electrons, which is intermediate between the binding energies of electrons for the $\mathrm{M}$ and $\mathrm{N}$ shells of uranium, 4.6 and $7.4 \mathrm{keV}$, respectively [33]. Thus, the injection energy of $10.8 \mathrm{MeV} / \mathrm{u}$ is the inflection point in terms of velocity matching.

Dependence of the distribution width $w_{q}$ on the target thickness would also exhibit the shell effect (Fig. 9). The measured dependence of $w_{q}$ on the thickness exhibits a dip around $0.7 \mathrm{mg} / \mathrm{cm}^{2}$ because of the charge compression at $64+$ due to the shell effect. The dip in the calculated distribution widths is small but shows the same tendency. This might indicate that the theory underestimates the difference between the $\mathrm{M}$ - and $\mathrm{N}$-shell ionization cross

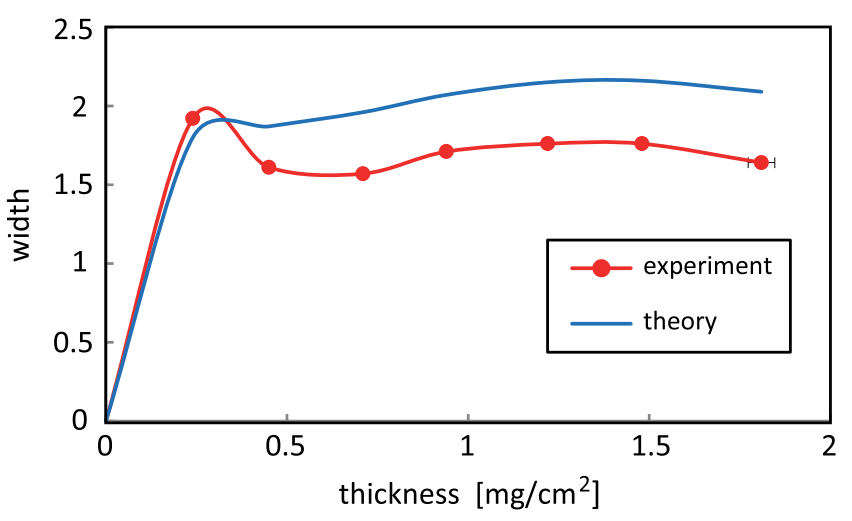

FIG. 9. Dependence of the charge-state distribution width $w_{q}$ on the target thickness.

sections. Further theoretical studies are required to explain these results.

\section{B. Energy loss and energy spread}

The ${ }^{238} \mathrm{U}$ beams passing through the He stripper (or carbon-foil strippers) were further transported and detected by the plastic scintillators (SC-H12, SC-H16, and SC-K51) located at the beam line before the IRC to measure the beam's kinetic energy and longitudinal distribution.

The distribution of the time difference between the signals of the scintillator and the bunch clock [29] were measured using two of the plastic scintillators, SC-H12 and SC-H16, respectively. The obtained time distributions were fitted with Gaussian functions to determine the time centers $\tau_{c}$. Using the two time centers for SC-H12 and SC-H16 $\left(t_{c}^{\mathrm{H} 12}\right.$ and $\left.t_{c}^{\mathrm{H} 16}\right)$, the mean time of flight $\tau_{\text {tof }}$ for the uranium beam can be determined as

$$
\tau_{\mathrm{tof}}=k \tau_{\mathrm{rf}}+\left(t_{c}^{\mathrm{H} 12}-t_{c}^{\mathrm{H} 16}\right)
$$

where $k$ and $\tau_{\text {rf }}$ are the wave number of the bunch clock and the clock period, respectively. The mean kinetic energy of the beam passing through the gas stripper, $E_{\text {exit }}$, can be calculated from $\beta=L / \tau_{\text {tof }} c$, where $L$ is the path length between SC-H12 and SC-H16 (10.0575 m). The obtained energies, which depend on the thickness of the He gas strippers and carbon-foil strippers, are shown in Table II.

The energy spread $\delta E / E_{\text {exit }}$ due to the strippers are derived as follows. First, the full width at half maximum (FWHM) of the obtained time distribution, $\tau_{\mathrm{FWHM}}^{K 51}(\xi)$, measured with SC-K51 was determined for all target thicknesses $\xi$. Then, the thickness dependence of $\tau_{\mathrm{FWHM}}^{K 51}(\xi)$ was linearly extrapolated to determine $\tau_{\mathrm{FWHM}}^{K 51}(\xi=0)$. The time of flight between the stripper

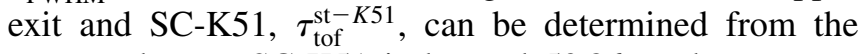
measured $E_{\text {exit }}$. SC-K51 is located $53.86 \mathrm{~m}$ downstream of the stripper exit. Finally, $\delta E(\xi) / E_{\text {exit }}$ is determined as 
TABLE II. Exit energy $E_{\text {exit }}$ after the strippers and longitudinal beam width $\tau_{\mathrm{FWHM}}^{K 51}$ for He gas measured with plastic scintillators (SC-H12 and SC-H16 for the energy and SC-K51 for the longitudinal profile). For comparison, the values measured with Arizona carbon-foil strippers are also listed. The charge state $q_{\mathrm{tr}}$ indicates the selected and transported charge state in the measurements.

\begin{tabular}{lcccc}
\hline \hline Stripper & Thickness $\left[\mathrm{mg} / \mathrm{cm}^{2}\right]$ & $q_{\mathrm{tr}}$ & $E_{\text {exit }}$ & $\tau_{\mathrm{FWHM}}^{K 51}$ \\
\hline Empty & $\ldots$ & $35+$ & $10.76(2)$ & $\ldots$ \\
$\mathrm{He}$ & $0.24(1)$ & $62+$ & $10.64(2)$ & $3.44(1)$ \\
& $0.47(1)$ & $65+$ & $10.51(2)$ & $3.80(1)$ \\
& $0.71(1)$ & $65+$ & $10.35(2)$ & $3.89(1)$ \\
& $0.97(2)$ & $65+$ & $10.22(2)$ & $4.06(1)$ \\
& $1.23(2)$ & $65+$ & $10.05(2)$ & $4.18(1)$ \\
& $1.51(3)$ & $65+$ & $9.90(2)$ & $4.43(1)$ \\
C foil & $1.83(4)$ & $65+$ & $9.70(2)$ & $4.72(1)$ \\
(Arizona) & $0.07(1)$ & $65+$ & $10.72(2)$ & $3.50(1)$ \\
& $0.28(1)$ & $71+$ & $10.61(2)$ & $4.16(1)$ \\
\hline \hline
\end{tabular}

$$
\frac{\delta E(\xi)}{E_{\text {exit }}}=\frac{\left.\sqrt{\left\{\tau_{\mathrm{FWHM}}^{K 5}(\xi)\right\}^{2}-\left\{\tau_{\mathrm{FWHM}}^{K 51}(0)\right.}\right\}^{2}}{\sqrt{2 \ln 2} \cdot \tau_{\mathrm{tof}}^{\mathrm{st}-K 51}} .
$$

The data for carbon-foil strippers (Arizona Carbon Foil Co.) were also analyzed by the same procedures for comparison.

The obtained energy spread for He and carbon foils are shown in Fig. 10. For comparison, the energy spread in $\mathrm{He}$ gas was calculated using the Monte Carlo method based on the scaled charge-changing cross sections derived in the previous subsection. In this calculation, the deposited charge-dependent partial energy $d E / d x\left(q_{i}, E\right)$, calculated

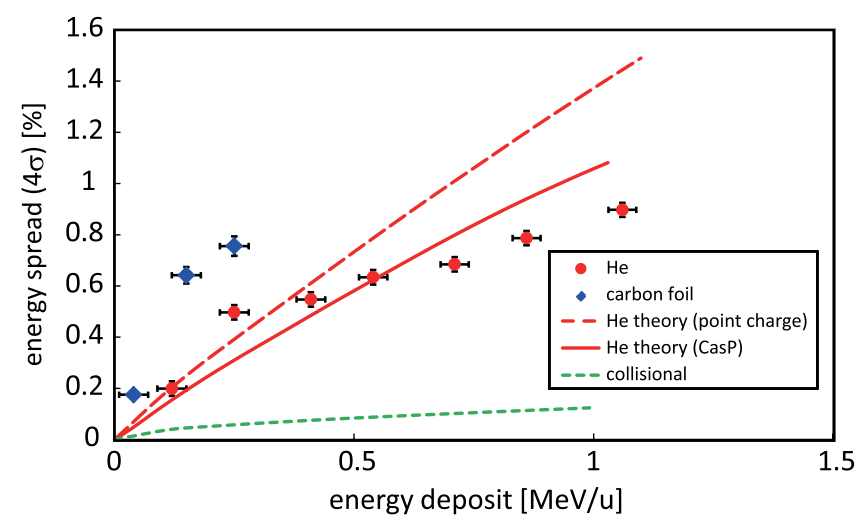

FIG. 10. Obtained energy spread for He and carbon-foil strippers as a function of energy deposit. Systematic errors due to $\tau_{w}^{K 51}(\xi=0)(\sim 5 \%)$ are excluded from the plots. Solid curves are based on calculations using the Monte Carlo method and the scaled charge-changing cross sections and energy-loss cross sections from the CASP code. The calculated energy spread for the point charges (dashed lines) and the calculation including only collisional energy spread (dotted lines) are also shown. using the CASP code [34], causes large energy spread ("charge-exchange" straggling). The energy spread for point-charge particles $\left(d E / d x\left(q_{i}, E\right) \propto q_{i}^{2}\right)$ was also calculated using Bethe's expression [38] with the corrections from the Bloch and Barkas terms [39,40] following Ref. [41]. The comparatively small effect of the "collisional" straggling, $\Omega_{c}^{2}$, which is based on Bohr's formulation [42], is also included in the calculation as

$$
d \Omega_{c}^{2}\left(q_{i}, E\right)=2 \pi \frac{m_{e}}{m_{u}} d E\left(q_{i}, E\right) \frac{E}{A} \frac{1}{\ln \left(T_{m} / I_{0}\right)},
$$

where $m_{e}$ is the electron mass, $m_{u}$ is the atomic mass unit, $T_{m}$ is the maximum energy transfer, and $I_{0}$ is the mean excitation energy of the target atom. This result indicates that the charge-exchange straggling is significant, which is discussed in Refs. [43,44].

\section{PERFORMANCE OF THE HE GAS STRIPPER FOR URANIUM BEAMS}

Two unfavorable features of the low- $Z$ gas stripper are its slow equilibration and large energy attenuation. These become serious concerns at high initial kinetic energies. Because the charge-changing cross sections for ${ }^{238} \mathrm{U}$ decrease at higher injection energies, thicker gas strippers are required to achieve the charge equilibrium state. Thus, the energy attenuation increases, which reduces the achievable maximum mean charge state. Figure 11 shows the calculated mean charge state as a function of the initial energy of ${ }^{238} \mathrm{U}^{35+}$ for various gas-stripper thicknesses. In this calculation, the scaled cross sections for He that were derived in the previous subsection were used. The calculated result shows the injection energy required to obtain the highest charge state for different gas thicknesses. A thickness of $2 \mathrm{mg} / \mathrm{cm}^{2}$ and an injection energy of at least $13 \mathrm{MeV} / \mathrm{u}$ are required to achieve a mean charge state of $69+$, which

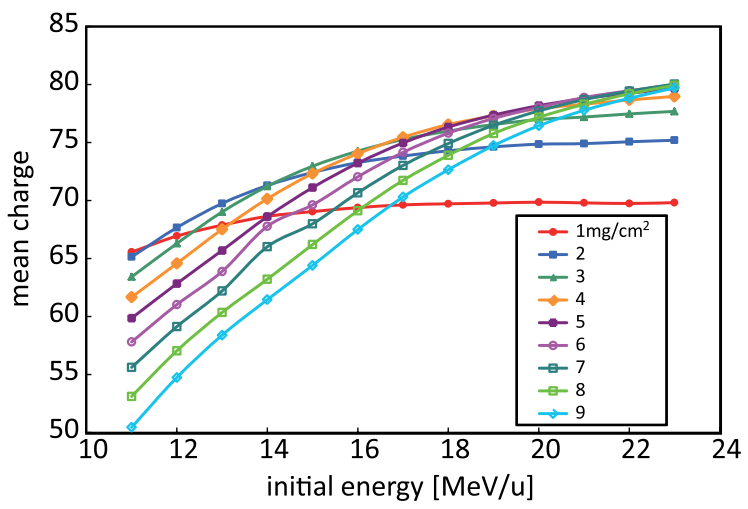

FIG. 11. Calculated dependence of the mean charge on the initial energy of ${ }^{238} \mathrm{U}^{35+}$ for several gas thicknesses. If we could prepare a $2-\mathrm{mg} / \mathrm{cm}^{2} \mathrm{He}$ gas stripper, the optimum injection energy of the uranium beams, which would reach the highest charge state, would be around $20 \mathrm{MeV} / \mathrm{u}$. This would yield a mean charge of approximately $75+$. 
is the minimum acceptable charge state in the $\mathrm{fRC}$ presently used at the RIBF. We require both a large accelerator to obtain $13 \mathrm{MeV} / \mathrm{u}$ and a matching large decelerator to achieve acceptable energy levels $(10.6 \mathrm{MeV} / \mathrm{u})$ for the fRC.

A better solution is to use thinner charge strippers and modify the fRC to match a lower charge state $(64-65+)$. In the present study, a $10.4-\mathrm{MeV} / \mathrm{u}{ }^{238} \mathrm{U}^{65+}$ beam was obtained using the $0.7-\mathrm{mg} / \mathrm{cm}^{2} \mathrm{He}$ stripper at a total beam transmission efficiency of $14 \%$, which is similar to the value for the current carbon-foil stripper.

\section{CONCLUSION}

For the first time, we developed and demonstrated an efficient charge-stripping system employing helium gas, which is used for charge-stripping high-intensity uranium beams.

We measured the charge-state evolution of ${ }^{238} \mathrm{U}$ beams injected at $10.8 \mathrm{MeV} / \mathrm{u}$ using the $\mathrm{He}$ gas stripper with a thickness of $0.24-1.83 \mathrm{mg} / \mathrm{cm}^{2}$. The obtained maximum mean charge state was approximately $65+$, which is considerably higher than that of medium- $Z$ gas strippers such as $\mathrm{Ne}$ and $\mathrm{Ar}$ (around 55+). The charge-state distribution was equivalent to the data obtained using the $8-\mathrm{m}$ target system, whereas the transmission efficiency was considerably higher than that of the 8-m system.

The scaling factors for the BEM and Schlachter's cross sections for He were both determined using a least squares fitting method with the measured charge-state distributions. These scaled cross sections reproduced the observed charge-state evolution in the He gas stripper.

The energy loss and energy spread due to the He gas stripper were also investigated. The experimentally determined and theoretical energy spread for the stripper were consistent.

A helium gas stripper having a thickness of around $0.7 \mathrm{mg} / \mathrm{cm}^{2}$ appears to be suitable as the first charge stripper for uranium beams at the RIBF. Further R\&D to determine its actual use are in progress in order to solve the problems associated with high gas consumption and heat load on the helium gas.

\section{ACKNOWLEDGMENTS}

The authors thank the operating crew for providing highquality beams and for their help during the preparation and measurements.

[1] Y. Yano, Nucl. Instrum. Methods Phys. Res., Sect. B 261, 1009 (2007).

[2] O. Kamigaito et al., in Proceedings of the 11th International Conference on Heavy Ion Accelerator Technology (Venezia, Italy, 2009), p. 21 [http://accelconf .web.cern.ch/AccelConf/HIAT2009/papers/mo-11.pdf].
[3] T. Nakagawa et al., High Energy Phys. Nucl. Phys. 31, 133 (2007); 79, 02A327 (2008)81, 02A320 (2010).

[4] K. Yamada et al., Report No. IPAC12, TUOBA02, 2012.

[5] Y. Higurashi, J. Ohnishi, T. Nakagawa, H. Haba, M. Tamura, T. Aihara, M. Fujimaki, M. Komiyama, A. Uchiyama, and O. Kamigaito, Rev. Sci. Instrum. 83, 02A308 (2012); 83, 02A333 (2012).

[6] M. Odera, Y. Chiba, T. Tonuma, M. Hemmi, Y. Miyazawa, T. Inoue, T. Kambara, M. Kase, T. Kubo, and F. Yoshida, Nucl. Instrum. Methods 227, 187 (1984).

[7] T. Mitsumoto, N. Fukunishi, A. Goto, N. Inabe, O. Kamigaito, H. Ryuto, N. Sakamoto, and Y. Yano, in Proceedings of the 17th International Conference on Cyclotrons and Their Applications (PASJ, Tokyo, Japan, 2004), p. 384.

[8] T. Mitsumoto et al., in Proceedings of the 16th International Conference on Cyclotrons and Their Applications (AIP, Melville, NY, 2001), p. 167.

[9] H. Okuno et al., IEEE Trans. Appl. Supercond. 18, 226 (2008).

[10] T. Kubo et al., Nucl. Instrum. Methods Phys. Res., Sect. B 204, 97 (2003).

[11] P. Thieberger et al., Phys. Rev. ST Accel. Beams 11, 011001 (2008).

[12] F. Marti, S. Hitchcock, O. Kester, and J. Oliva, in Proceedings of the 25th International Linear Accelerator Conference (Tsukuba, Ibaraki, Japan, 2010), p. 659 [http:// accelconf.web.cern.ch/AccelConf/LINAC2010/papers/tup 105.pdf].

[13] W. Barth, in Proceedings of the 24th International Linear Accelerator Conference (Victoria, BC, Canada, 2008), p. 31 [http://accelconf.web.cern.ch/AccelConf/LINAC08/ papers/mo204.pdf].

[14] H. Ryuto, H. Hasebe, N. Fukunishi, T. Abe, A. Goto, M. Kase, and Y. Yano, Nucl. Instrum. Methods Phys. Res., Sect. A 581, 586 (2007).

[15] N. Bohr and J. Lindhard, K. Dan. Vidensk. Selsk. Mat. Fys. Medd. 28, 7 (1954).

[16] H. D. Betz and L. Grodzins, Phys. Rev. Lett. 25, 211 (1970).

[17] N. Fukunishi et al., in Proceedings of the 23rd Particle Accelerator Conference, Vancouver, Canada, 2009 (IEEE, Piscataway, NJ, 2009), MO3GRI01.

[18] H. Hasebe, H. Okuno, H. Kuboki, H. Ryuto, N. Fukunishi, O. Kamigaito, A. Goto, M. Kase, and Y. Yano, Nucl. Instrum. Methods Phys. Res., Sect. A 613, 453 (2010).

[19] H. Hasebe, H. Kuboki, H. Okuno, N. Fukunishi, O. Kamigaito, H. Imao, A. Goto, and M. Kase, Nucl. Instrum. Methods Phys. Res., Sect. A 655, 57 (2011).

[20] H. Kuboki, H. Okuno, S. Yokouchi, H. Hasebe, T. Kishida, N. Fukunishi, O. Kamigaito, A. Goto, M. Kase, and Y. Yano, Phys. Rev. ST Accel. Beams 13, 093501 (2010).

[21] H. Kuboki et al., Phys. Rev. ST Accel. Beams 14, 053502 (2011).

[22] H. Okuno et al., Phys. Rev. ST Accel. Beams 14, 033503 (2011).

[23] H. D. Betz, Rev. Mod. Phys. 44, 465 (1972).

[24] R. E. Olson, R. L. Watson, V. Horvat, A. N. Perumal, Y. Peng, and Th. Stöhlker, J. Phys. B 37, 4539 (2004).

[25] R. E. Olson, R. L. Watson, V. Horvat, K. E. Zaharakis, R. D. DuBois, and Th. Stöhlker, Nucl. Instrum. Methods Phys. Res., Sect. A 544, 333 (2005). 
[26] V.P. Shevelko, Th. Stöhlker, H. Tawara, I. Yu. Tolstikhina, and G. Weber, Nucl. Instrum. Methods Phys. Res., Sect. B 268, 2611 (2010).

[27] T. Kishida et al., Nucl. Instrum. Methods Phys. Res., Sect. A 438, 70 (1999).

[28] H. Imao et al., RIKEN Accel. Prog. Rep. 44, 116 (2010).

[29] T. Watanabe et al., RIKEN Accel. Prog. Rep. 39, 241 (2005).

[30] M. Gryzinski et al., Phys. Rev. 138, A305 (1965).

[31] A.S. Schlachter, J. Stearns, W. Graham, K. Berkner, R. Pyle, and J. Tanis, Phys. Rev. A 27, 3372 (1983).

[32] J. H. McGuire and P. Richard et al., Phys. Rev. A 8, 1374 (1973).

[33] K. Rashid, M.Z. Saadi, and M. Yasin, At. Data Nucl. Data Tables 40, 365 (1988).

[34] P. L. Grande and G. Schiwietz, CASP ver. 1.4 [http://www.helmholtz-berlin.de/people/gregor-schiwietz/casp_en .html].
[35] P.L. Grande and G. Schiwietz, Phys. Rev. A 58, 3796 (1998).

[36] G. Schiwietz and P. L. Grande, Nucl. Instrum. Methods Phys. Res., Sect. B 153, 1 (1999).

[37] F. James and M. Ross, MINUIT Users Guide, Program Library D506, CERN (1981).

[38] H. Bethe, Handb. Phys. 24, 273 (1933).

[39] F. Bloch, Ann. Phys. (Leipzig) 16, 285 (1933).

[40] W. H. Barkas, J. Dyer, and H. Heckman, Phys. Rev. Lett. 11, 26 (1963).

[41] B. A. Weaver, and A. J. Westphal, Nucl. Instrum. Methods Phys. Res., Sect. B 187, 285 (2002).

[42] N. Bohr, Philos. Mag. 30, 581 (1915).

[43] O. Vollmer, Nucl. Instrum. Methods 121, 373 (1974).

[44] H. Weick et al., Phys. Rev. Lett. 85, 2725 (2000). 